\title{
Meio ambiente e saúde: metodologia para análise espacial da ocorrência de malária em projetos de assentamento
}

\author{
Marcia Caldas de Castro* \\ Burton Herbert Singer ${ }^{* *}$
}

\begin{abstract}
A transmissão de malária em projetos de assentamento na Amazônia, definida como malária de fronteira, é resultado de um intrincado processo envolvendo fatores biológicos, ecológicos, socioeconômicos e comportamentais, apresentando uma transição temporal de altas a baixas taxas ao longo de aproximadamente oito anos. Como resultado, um dos grandes desafios é a compreensão desse processo, através da identificação das variáveis determinantes da transmissão, considerando-se dimensões temporais e espaciais. Neste artigo é apresentada uma abordagem metodológica que caracteriza perfis de risco de malária em projetos de assentamento, a partir de uma análise multidisciplinar. Composta de três etapas, a abordagem combina análise espacial, geoestatística e modelos de Grade of Membership. Os resultados ressaltam a importância de medidas de controle diferenciadas de acordo com o estágio do projeto de assentamento (implementação recente ou antiga) e o nível de transmissão em cada localidade.
\end{abstract}

Palavras-chave: Malária. Projetos de colonização. Amazônia. Krigagem. Indicadores locais de associação espacial. Modelos de Grade of Membership.

\section{Introdução}

A malária é provavelmente tão antiga quanto a espécie humana, porém sua etiologia só foi descoberta no final do século XIX. Em 1898, Ronald Ross provou que o vetor de transmissão era o mosquito. Alguns anos antes, em 1880, Charles-Louis-Alphonse Laveran foi o primeiro a identificar o parasita da malária no microscópio (DESOWITZ, 1991). Em 1820, o quinino começou a ser produzido em larga escala, permanecendo como única droga para tratamento da malária por cerca de 110 anos (BOYD, 1949; JARCHO; TORTI, 1993). Em 1905, Carlos
Chagas estabeleceu a importância das condições da habitação para a transmissão da doença, sugerindo o uso de telas e a fumigação das casas pelo vapor de enxofre (CHAGAS, 1934; SILVEIRA; REZENDE, 2001). Pela primeira vez foi caracterizado o conceito de malária como doença domiciliar, raramente adquirida no exterior do domicílio, e foi adotada medida visando a eliminação do mosquito transmissor (DEANE, 1986). Em 1940 Paul Müller patenteou o DDT que permite eliminar mosquitos presentes no interior de domicílios através da borrifação (DESOWITZ, 1991). Com base nesse conhecimento adquirido desde o final do

\footnotetext{
* Professora assistente de Demografia do Department of Population and International Health, Harvard School of Public Health.

** Professor titular de Demografia e Políticas Públicas, Princeton University.
} 
século XIX, medidas tradicionais de controle da doença incluem: uso de larvicidas; borrifação do interior de domicílios; modificação ambiental (drenagem, por exemplo); uso de telas e mosquiteiros; e uso de repelentes (BRUCE-CHWATT, 1987; NÁJERA et al., 1992).

Apesar de todo esse conhecimento disponível, a malária atinge aproximadamente 500 milhões de pessoas no mundo, causando mais de um milhão de mortes. $\mathrm{Na}$ Amazônia, mais de 600 mil casos da doença foram registrados em 2005 , o que representa um aumento de $29,4 \%$ em relação ao ano anterior (MINISTÉRIO DA SAÚDE, 2006). Embora não resulte em elevado número de mortes, a morbidade é alta, trazendo sérias conseqüências socioeconômicas. O controle da doença na Amazônia, entretanto, é um desafio, uma vez que medidas tradicionais de controle não são eficazes. Isto porque a transmissão ocorre majoritariamente no peridomicílio, o mosquito pica preferencialmente das 16 às 18 e das 5 às 6 horas, modificações ambientais favorecem a formação de criadouros de mosquito e o conhecimento e comportamento individuais maximizam a exposição ao risco de transmissão (SAWYER; SAWYER, 1987). Desta forma, é necessário um detalhado conhecimento dos fatores que determinam a transmissão da doença, a fim de que medidas alternativas possam ser planejadas e implementadas. Tal conhecimento demanda uma análise multidisciplinar, permitindo incorporar questões biológicas, ecológicas, socioeconômicas e comportamentais e considerando a existência de possíveis variações temporais e espaciais. Este artigo apresenta uma abordagem metodológica que possibilita a realização dessa análise multidisciplinar.

\section{Meio ambiente e malária}

Fatores ambientais desempenham um papel importante no risco de transmissão de malária, podendo ser divididos em dois grupos: meio ambiente natural e meio ambiente modificado pela ação humana. $\mathrm{O}$ impacto de cada um desses grupos varia por área geográfica e depende do contexto socioeconômico em que são observados. Considerando o contexto Amazônico, o meio ambiente natural inclui topografia, hidrografia, tipo de solo e clima. Estudos realizados no final do século XIX e início do XX já deixavam claro a importância de cada um desses fatores na transmissão da malária. $A$ floresta Amazônica oferece condições ideais de temperatura e umidade para o ciclo de vida do mosquito (SAWYER; SAWYER, 1987; SAWYER, 1992). Além disso, o padrão pluviométrico maximiza o risco de transmissão no início e final da estação chuvosa, quando os solos estão mais úmidos e a variação do nível da água nos rios e igarapés propicia a formação de criadouros de mosquito em suas margens (CRUZ, 1910; PEIXOTO, 1917). De fato, dados empíricos revelam a alta exposição de populações ribeirinhas à malária (CRUZ, 1913; WAGLEY, 1964). Além disso, solos argilosos e baixas altitudes favorecem a transmissão da doença (HIRSCH, 1883).

Se as características descritas anteriormente, por um lado, facilitam a transmissão de malária, por outro, não são fatores que por si só determinam a ocorrência da doença. É através da ação do homem, transformando o meio ambiente natural, que questões sanitárias são observadas com diferentes graus de gravidade $(\mathrm{HIRSCH}$, 1883; WAGLEY, 1964). O meio ambiente modificado pela ação do homem inclui a qualidade da habitação e do local onde a moradia é estabelecida (por exemplo, distância entre a casa e a floresta, igarapés, rios e estrada).

Tendo em vista a dinâmica de projetos de assentamento na Amazônia, em geral a transformação ambiental é intensa. Inicialmente, são abertas estradas na mata e, logo após a ocupação do lote, uma pequena área é desmatada pelos parceleiros, dando lugar à moradia e agricultura. Progressivamente, áreas adicionais são desmatadas a fim de que a produção agrícola seja estabelecida. Vários problemas surgem a partir dessa transformação ambiental. Em primeiro lugar, a qualidade da moradia é pobre, uma vez que os parceleiros não dispõem de recursos financeiros adequados. Os materiais usados incluem plástico, papelão, compensados 
e folhas de palmeira. A casa não oferece proteção contra mosquitos e torna inviável o uso de borrifação intradomiciliar. Outro problema é a qualidade do desmatamento e o tempo que a terra desmatada fica exposta. $\mathrm{O}$ número de criadouros de mosquito aumenta significativamente e o contacto entre 0 homem e o mosquito torna-se mais intenso. Exemplos de associação entre malária e meio ambiente na Amazônia incluem áreas de garimpo (SCHMINK, 1985; COIMBRA JR., 1988; ARON; PATZ, 2001) e projetos de infra-estrutura (FEARNSIDE, 1989; IÑIGUEZ ROJAS; TOLEDO, 1998; FEARNSIDE, 1999; GUIMARÃES et al., 2004), além de projetos de assentamento (MARQUES, 1987; SINGER; CASTRO, 2001).

\section{Expansão da fronteira Amazônica e incidência de malária}

Nos últimos 40 anos, a expansão da fronteira Amazônica assumiu proporções significativas a partir da implantação de projetos centrados em agricultura, extração mineral, criação de gado e fixação do homem à região (MORAN, 1985; SCHMINK; WOOD, 1992; BROWDER; GODFREY, 1997). Baseados em ideais geopolíticos, esses projetos tinham como objetivo integrar a Amazônia com as demais regiões do país e garantir a segurança nacional (SILVA, 1967). $\mathrm{Na}$ prática, foi registrado intenso fluxo migratório, principalmente de pessoas de baixo poder econômico advindas das regiões Sul e Nordeste (MARTINE, 1990). Além disso, importantes e significativas transformações ecológicas foram observadas (desmatamento, construção de estradas e hidrelétricas, etc.), contribuindo para um crítico aumento do número de casos de malária (COIMBRA, 1985; MARQUES, 1987).

A população da Amazônia cresceu de 7,2 milhões em 1970 para 21,1 milhões em 2000. Em 1970 pouco mais de 50 mil casos de malária foram registrados no Brasil (DEANE, 1988). Em 1982 esse número já era da ordem de 200 mil, atingindo meio milhão de casos em 1988. O Estado de Rondônia, que concentrou um grande número de projetos de assentamento, contribuiu com $43 \%$ dos casos de malária registrados no país e 46\% daqueles encontrados na Amazônia, em 1986, ficando conhecido como a "capital da malária" no Brasil. Em 2004, entre os dez municípios com Índice Parasitário Anual (IPA) ${ }^{1}$ mais elevado, seis pertenciam ao Estado de Rondônia. Atualmente, cerca de $99 \%$ dos casos de malária registrados no Brasil concentram-se na Amazônia. Dos poucos casos observados em outras regiões do país, $64 \%$ são importados (55\% provêm da Amazônia). Em 2005, mais de 600 mil casos de malária foram registrados na Amazônia, representando um crescimento de $29,4 \%$ em relação ao ano anterior (MINISTÉRIO DA SAÚDE, 2006).

Um entendimento detalhado da expansão de malária na Amazônia demanda uma análise multidisciplinar, combinando aspectos biológicos e ecológicos em diferentes escalas, com questões socioeconômicas e comportamentais. Nesse sentido, Castro et al. (2006a) expandiram o conceito de malária de fronteira, inicialmente proposto por Sawyer (1988; 1992), em três níveis hierárquicos. O primeiro representa uma escala microindividual e incluindo os seguintes fatores de risco: alta densidade de Anopheles darlingi, o principal mosquito transmissor de malária na Amazônia, em conseqüência de mudanças ambientais que favorecem a proliferação de criadouros (TADEl et al., 1988); intensa exposição humana, resultado do conhecimento limitado dos modos de transmissão da doença, do padrão comportamental da atividade de picada do mosquito transmissor, que pica mais intensamente no peridomicílio entre 18 e 20 horas e das 5 às 6 horas (LOURENÇODE-OLIVEIRA, et al., 1989; KLEIN; LIMA, 1990); a taxa de mortalidade devido à malária é baixa, mas a taxa de morbidade é elevada, resultando em significativos im-

\footnotetext{
${ }^{1}$ O Índice Parasitário Anual representa o número de exames positivos por 1.000 habitantes. O Ministério da Saúde considera alto risco de transmissão os municípios que registram IPA maior ou igual a 50 . Os dez municípios com maior IPA em 2004 registraram índices entre 294 e 502.
} 
pactos sociais e econômicos; os níveis de imunidade adquirida são baixos entre os parceleiros, uma vez que grande parte veio de regiões onde a malária não é observada; a moradia é de baixa qualidade, inviabilizando o uso de borrifação domiciliar; e os serviços locais de saúde são deficientes. O segundo nível representa a comunidade, sendo que a malária de fronteira é caracterizada por frágeis instituições privadas e governamentais, ausência de esforços comunitários organizados, marginalidade política e alta mobilidade populacional. Tais características tornam difícil a concretização de esforços locais para promoção de manejo ambiental e redução de transmissão de malária. Finalmente, o terceiro é o nível estadual e nacional, em que malária de fronteira é caracterizada por áreas assentadas sem um apropriado planejamento, muitas vezes resultantes de ocupação ilegal por parceleiros que não tiveram sucesso em outras áreas.

Além de não ter uma conceituação única, a malária de fronteira também apresenta evolução temporal específica caracterizada por três fases, conforme sugerido por Sawyer e Sawyer (1992). A primeira fase, durante os anos iniciais do projeto de assentamento, é marcada por um rápido aumento da incidência de malária, em que são observadas as características que definem os dois primeiros níveis do conceito de malária de fronteira. A segunda fase, que ocorre aproximadamente três anos após a abertura do assentamento, dura cerca de cinco anos e é marcada por um declínio progressivo das taxas de malária, acompanhando uma esperada melhoria das condições ambientais e socioeconômicas. Finalmente, a terceira etapa é caracterizada por um cenário estável com baixas taxas de malária, resultado do rápido processo de urbanização, da formação de um senso comunitário, da melhoria das condições socioeconômicas e de uma redução das mudanças ambientais observadas nos anos iniciais de ocupação.

A duração de cada fase pode variar, dependendo do processo de consolidação do projeto de assentamento e das ações de controle de saúde implementadas.
Entretanto, independentemente da transição temporal observada, fica claro que a escolha e a implementação de medidas mitigadoras devem considerar o estágio em que se encontra o projeto de assentamento, bem como o fato de que a importância dos diversos determinantes de transmissão de malária varia em cada uma das três fases do proces-so de transição. Portanto, identificar esses determinantes é crucial para o planejamento de programas de controle.

\section{Caracterização de áreas de risco}

Pelo descrito anteriormente, fica claro que a interação homem, meio ambiente e saúde observada na Amazônia, em especial nos projetos de assentamento, é complexa. O estudo e a compreensão dos determinantes da incidência de malária em áreas de fronteira demandam abordagens inovadoras que permitam uma análise multidisciplinar, devendo considerar, ainda, possíveis efeitos espaciais, assim como altos níveis de heterogeneidade nos dados. A seguir é apresentada uma proposta de abordagem metodológica, composta de três etapas distintas, cujo objetivo é identificar os determinantes da transmissão de malária em projetos de colonização, caracterizando perfis de risco sob uma perspectiva espacial e temporal. Cada etapa utiliza uma modelagem distinta, combinando análise estatística espacial, geoestatística e modelos de classificação nebulosa (CASTRO, 2002).

A primeira etapa consiste em avaliar se a transmissão de malária apresenta correlação espacial e se áreas com alta ou baixa concentração de casos podem ser identificadas. São utilizados indicadores locais de associação espacial (ANSELIN, 1995), especificamente o teste $G_{i}^{*}(d)$, recomendado para identificação de conglomerados significativos de valores altos ou baixos próximos a uma localidade $i$, dada uma distância $d$ de $i$ (GETIS; ORD, 1996). No caso de projetos de assentamento, a localidade $i$ refere-se a um lote e a distância $d$ define a área de vizinhança de cada lote. É esperado que lotes próximos apresentem taxas de malária semelhantes (Primeira Lei da Geografia de Tobler, 1979). Valores padronizados do teste 
$\mathrm{G}_{i}{ }^{*}(d)$ são comparados com a distribuição estatística normal, a fim de identificar a presença de conglomerados significativos (GETIS; ORD, 1992; ORD; GETIS, 1995).

Duas questões devem ser analisadas com relação ao uso do teste $\mathrm{G}_{i}{ }^{*}(d)$ : comparações múltiplas e dependentes; e escolha da distância $d$. A primeira decorre do fato de que, para cada localidade $i$, é aplicado um teste, sendo que essa multiplicidade pode resultar em erros do tipo I elevados (KURTZ et al., 1965; TUKEY, 1991). Além disso, diferentes localidades possuem vizinhanças semelhantes e, portanto, os testes não são independentes (ANSELIN, 1995; GETIS; ORD, 1996; ROGERSON, 2001). Esse problema é normalmente contornado a partir do uso da correção de Bonferroni para a multiplicidade (GETIS; ORD, 2000). Entretanto, uma alternativa mais eficiente e robusta é o uso da taxa de falsas descobertas, proposta por Benjamini e Hochberg (1995), que controla a taxa média de erros causados pela falsa rejeição da hipótese nula. A aplicação desse ajuste é extremamente simples e pode ser implementada em planilhas eletrônicas. Com base em dados empíricos e simulados, Castro e Singer (2006) mostram o ganho significativo obtido com o uso da taxa de falsas descobertas em aplicações de testes locais de associação espacial.

A segunda questão - escolha da distância $d$ - é conceitualmente mais complexa. Não há uma regra preestabelecida para sua escolha, e tampouco procedimentos estatísticos que ajudem sua definição. Embora diferentes critérios tenham sido utilizados (por exemplo, GETIS, 1995; PAEZ et al., 2001; BAUMONT et al., 2004), cada caso deve ser analisado com cautela. Idealmente, a distância deve ser escolhida com base nas características físicas da área e nas particularidades do fenômeno em estudo.

Essa primeira etapa da abordagem metodológica pode ser aplicada em tempo real, facilitando o monitoramento de focos epidêmicos que demandam ação imediata por parte de serviços locais de saúde. Pode ser utilizada, ainda, em dados históricos, revelando padrões locais e a forma como estes se modificam ao longo do tempo. Em ambos os casos, conglomerados significativos são identificados e localizados espacialmente. A presença de conglomerados sugere a ocorrência de processos distintos em certas localidades, cuja compreensão permite a adoção de medidas alternativas de controle da doença. Ou seja, sugere que a área em estudo poderia ser dividida em subáreas (agrupamentos de lotes) a serem analisadas em detalhe através de um estudo multivariado.

A segunda etapa da abordagem metodológica facilita a definição das subáreas anteriormente mencionadas. Nessa etapa, estimativas espaciais das taxas de malária são calculadas para todos os lotes do assentamento em estudo, utilizandose o método geoestatístico de krigagem (ISAAKS; SRIVASTAVA, 1989; CRESSIE, 1991), que estima o valor de uma variável em localidades não amostradas com base nas amostras vizinhas. A estrutura espacial dos dados observados é representada pelo semivariograma e depende apenas da localização de cada variável e da distância que as separa (CRESSIE, 1991). Através de funções matemáticas, essa estrutura espacial permite o cálculo de pesos que, multiplicados pelos valores observados, resultam em estimativas para os pontos não amostrados. Conforme a Primeira Lei da Geografia (TOBLER, 1979), esses pesos são maiores para localidades próximas e menores para aquelas distantes. Cressie (1991) apresenta detalhes do modelo de krigagem e dos procedimentos de estimação.

Os resultados da primeira etapa (identificação de conglomerados nas taxas de malária observadas nos lotes amostrados), combinados com os da segunda etapa (estimativas espaciais dessas taxas para a totalidade dos lotes), possibilitam a definição de subáreas de risco de malária. É esperado que uma análise multivariada dessas subáreas revele especificidades do processo de transmissão da doença, tarefa a ser executada na terceira e última etapa da abordagem metodológica, em que modelos de Grade of Membership (GoM), baseados em métodos de classificação nebulosa (SINGER, 1989; MANTON et al., 1994) são aplicados em cada subárea, revelando os determinantes 
de altos e baixos riscos de transmissão. Esses modelos são especialmente úteis na análise de populações heterogêneas, em que combinações de ocorrências de variáveis não são registradas com elevada freqüência. $A$ cada indivíduo da população é atribuído um conjunto de escores $g$ que indicam seu grau de pertinência em partições difusas ou perfis. Considerando um cenário com dois perfis, alto e baixo risco de malária, cada indivíduo teria dois escores representando sua similaridade com cada perfil, sendo que a soma desses escores é igual a unidade. Um escore $g=1$ para o perfil de alto risco indica que o indivíduo possui todas as características que descrevem esse perfil. Um indivíduo pode, ainda, apresentar características de ambos os perfis - por exemplo, se $g=3 / 4$ para o perfil de alto risco, esse indivíduo apresenta um grau de similaridade mais elevado com o perfil de alto risco, ainda que $1 / 4$ de seus atributos caracterize um perfil de baixo risco (Castro et al., 2006a).

A descrição e a análise desses perfis para cada subárea facilitam o entendimento do processo de transmissão de malária, a identificação de especificidades locais e a elaboração de propostas inovadoras para o controle da doença. Berkman et al. (1989), Manton et al. (1994) e Erosheva (2003) apresentam detalhes do processo de estimação do modelo de GoM.

A seguir é detalhada uma aplicação da abordagem metodológica aqui descrita. Como estudo de caso, foi selecionado o projeto de assentamento de Machadinho, localizado no Estado de Rondônia.

\section{Estudo de caso: Machadinho, Rondônia}

O projeto de colonização de Machadinho foi implantado em 1984 como parte do Polonoroeste - Programa Integrado de Desenvolvimento do Noroeste do Brasil. Planejado para ser um modelo de colonização sustentável, Machadinho teve um traçado inovador, em que lotes foram definidos com base na topografia e hidrologia locais. Antes da implantação, a área do projeto era mata fechada, esparsamente ocupada por seringueiros. Acredita-se que esses seringueiros eram portadores do parasita da malária, porém assintomáticos, e sua presença não foi formalmente considerada durante a fase de planejamento e distribuição dos lotes, uma vez que residiam em áreas designadas como reservas florestais.

A ocupação de Machadinho foi feita por migrantes que vieram majoritariamente do Sul do Brasil, região livre de malária. Esses migrantes não dispunham de conhecimento adequado sobre os meios de transmissão de malária e sobre formas de proteção individual, além de não possuírem imunidade adquirida. Parte dos migrantes se estabeleceu temporariamente em áreas malarígenas em Rondônia, aguardando o momento de ocupar seus lotes.

A ocupação inicial do lote é marcada por derrubada de parte da mata, a fim de que o parceleiro possa construir sua casa (em princípio temporária) e iniciar a produção agrícola. Essa derrubada é, na maioria dos casos, precária e lenta, favorecendo a proliferação de criadouros de mosquito. A presença de indivíduos assintomáticos, combinada com a introdução de população não imune e o aumento do número de mosquitos, resultou na ocorrência de graves surtos de malária, fato que foi registrado na maioria dos projetos de assentamento implantados na Amazônia.

Em Machadinho, o IPA alcançou 3.400 slides positivos por 1.000 pessoas em $1985 ; 65,7 \%$ da população teve pelo menos um episódio de malária no mesmo ano e $90,1 \%$ no ano seguinte. Em 1986, 55,9\% da população de Machadinho registrou um episódio da doença em mais de cinco meses ao longo do ano (SAWYER; SAWYER, 1987; SYDENSTRICKER, 1992).

A transmissão de malária em Machadinho reúne todas as condições anteriormente descritas como malária de fronteira. A busca eficaz de medidas alternativas de controle da doença reside no entendimento de seus determinantes, sua diferenciação espacial e sua evolução ao longo do tempo. A abordagem metodológica apresentada anteriormente possibilita esse entendimento, como é mostrado a seguir.

Pesquisas de campo foram realizadas nos lotes ocupados das glebas 1 e 2 de Machadinho em 1985, 1986, 1987 e 1995 (a 
Figura 1 apresenta a localização geográfica do projeto). Considerou-se o lote ocupado quando o parceleiro residia no local e já havia iniciado o desmatamento (SAWYER, 1985). Desta forma, não foram incluídos na pesquisa lotes onde a derrubada já havia sido iniciada, mas o parceleiro não havia estabelecido residência. O questionário utilizado teve caráter abrangente, coletando informações demográficas, socioeconômicas, ecológicas, de saúde e práticas agrícolas (SAWYER, 1985; SAWYER; SAWYER, 1987).

Casos de malária foram declarados pelos parceleiros para cada um dos 12 meses precedentes à pesquisa. As vantagens e limitações do uso dessa informação são discutidas em detalhe por Castilla e Sawyer (1993), Sawyer e Sawyer (1987) e Singer e Sawyer (1992). Taxas de exposição à malária foram calculadas considerando-se os casos declarados mensalmente e a localidade onde a pessoa se encontrava em cada mês.
A taxa varia entre zero e um; o primeiro valor indica ausência de episódios de malária ao longo do ano, enquanto o segundo mostra que a pessoa declarou a ocorrência de casos em todos os meses do ano. A distribuição geográfica da taxa é extremamente diversa, sendo que valores elevados tendem a ser observados com maior freqüência na gleba 1 de Machadinho.

Variáveis adicionais foram construídas a partir do uso de sistemas de informação geográfica (SIG) - por exemplo, distância do lote ao posto de saúde, lotes localizados a menos de $1 \mathrm{~km}$ da reserva florestal, distância do lote ocupado mais próximo, entre outras (CASTRO, 2002). Além disso, foram utilizadas informações etnográficas, coletadas de 1985 a 1995 (Castro et al., 2006a). Finalmente, imagens de satélite (Landsat $\mathrm{TM}$, com resolução de $30 \mathrm{~m}$ ) capturadas em 1985, 1986, 1994 e 1995 possibilitaram a identificação de fatores adicionais de risco,

FIGURA 1

Localização espacial e características físicas do Projeto de Assentamento de Machadinho
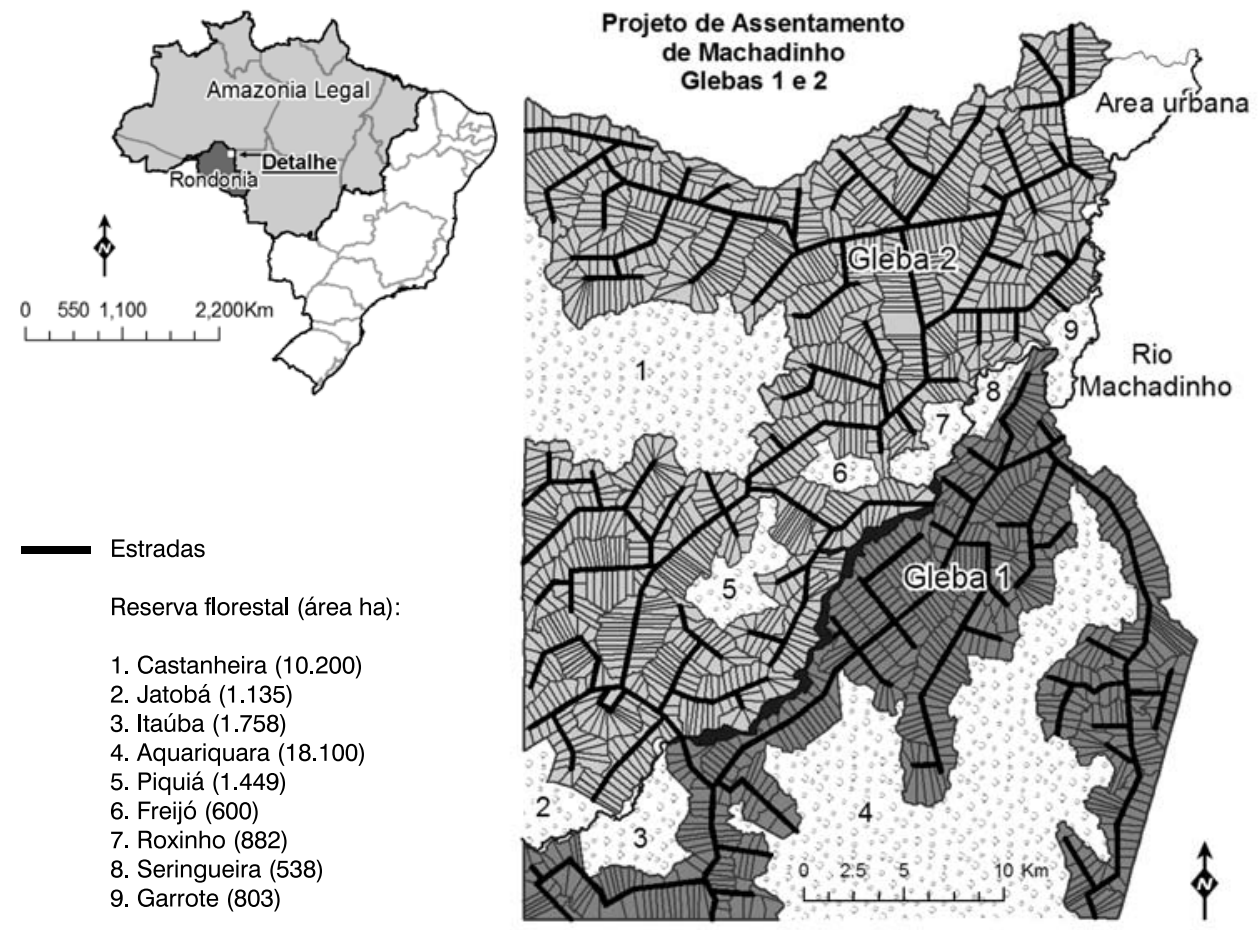

Fonte: Incra. 1982. Anteprojeto de assentamento Machadinho (Rondônia). Brasília: Incra/DP/Ceer. 
não coletados nas pesquisas de campo (CASTRO et al., 2006a).

As duas primeiras etapas da abordagem metodológica descrita neste artigo utilizam apenas as taxas de malária. $O$ teste $G_{i}^{*}(d)$ foi calculado usando-se uma distância de $3.500 \mathrm{~m}$, selecionada com base em três critérios. O primeiro refere-se às características de distância de vôo do Anopheles darlingi, principal mosquito transmissor de malária na Amazônia (DEANE, 1947; COVA-GARCIA, 1961; VAN THIEL, 1962; TADEl et al., 1998). O segundo diz respeito às características físicas dos lotes em Machadinho (desenho irregular e tamanho médio) e suas implicações na definição da área de vizinhança no entorno de cada lote (CASTRO et al. , 2006b). Finalmente, o terceiro critério considera a abrangência geográfica dos setores - unidade espacial utilizada pelos serviços locais de saúde (CASTRO et al., 2006b).

Para o cálculo do teste $\mathrm{G}_{i}^{*}(d)$, foi utilizado o software PPA - Point Pattern Analysis (ALDSTADT et al., 2007). A Figura 2 mostra os resultados do teste, revelando a presença de aglomerações significativas de altas e baixas taxas de malária nas glebas 1 e 2 de Machadinho (a multiplicidade de testes foi corrigida pela taxa de falsas descobertas). É interessante notar que, apesar da elevada transmissão de malária observada logo após o processo inicial de ocupação, aglomerações de baixas taxas de malária foram identificadas ao longo dos anos. É impera-

FIGURA 2

Aglomerados de altas e baixas taxas de malária, segundo o teste $G^{*}{ }_{i}(d)$ Machadinho - 1985-1995
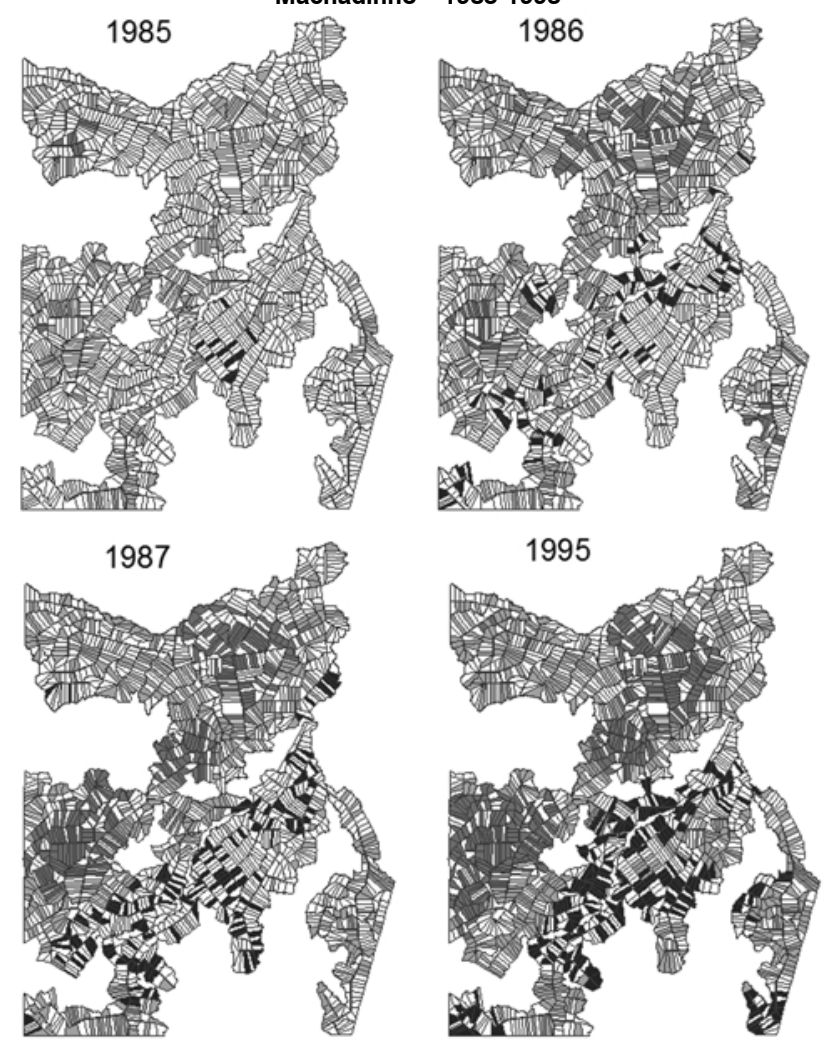

Lotes com aglomeraçāo significativa de baixas taxas de malária Lotes sem aglomeração significativa de taxas de malária Lotes com aglomeração significativa de altas taxas de malária Lotes não amostrados 
tivo analisar detalhadamente esses casos, uma vez que lições importantes podem ser aprendidas e incorporadas em campanhas de controle. Esses resultados revelam, ainda, a importância de se incluir um componente espacial na análise e evidenciam a importância de campanhas de controle direcionadas às áreas críticas (AKHAVAN et al., 1999; CARTER et al., 2000). Finalmente, é possível observar que os conglomerados identificados não oferecem informação suficiente para a partição da área total em subáreas de risco. O ideal é que se disponha de informações sobre cada lote, de tal forma que essas subáreas possam ser traçadas. Esse problema é solucionado na segunda etapa da abordagem metodológica.

Utilizando o método de krigagem foram obtidas estimativas espaciais para as taxas de malária em todos os 1.472 lotes das glebas 1 e 2 de Machadinho. Com base nessas estimativas e na localização dos conglomerados, subáreas foram definidas - três em 1985 e 1995, quatro em 1986 e cinco em 1987, conforme mostra a Figura 3. Para cada uma dessas subáreas foi aplicado o método de GoM.

O objetivo desta terceira etapa é identificar combinações de ocorrências de variáveis que estejam associadas a um perfil de alto ou baixo risco de malária. Para tanto, as informações disponíveis em cada ano foram divididas em dois grupos: meio ambiente e comportamento/economia. Três categorias podem ser identificadas em cada grupo: qualidade habitacional, proximidade de áreas críticas (mata, riacho, água parada, etc.) e área desmatada, no primeiro grupo; e nível educacional, história migratória e características econômicas, no segundo (CASTRO et al., 2006a).

Em cada categoria, a significância da ocorrência das variáveis na determinação dos perfis é avaliada. A partir daí, a contribuição de cada um dos dois grupos para a definição dos perfis de alto ou baixo risco de malária é considerada: alta, caso uma ou mais ocorrências em cada uma das três categorias do grupo forem significativas; média, se uma ou mais ocorrências em duas das três categorias do grupo forem significativas; e baixa, caso uma ou mais ocorrências em uma das três categorias forem significativas.

A análise dos perfis destaca importantes aspectos do processo de transmissão de malária em projetos de assentamento. Primeiro, os resultados revelam que nos anos iniciais do projeto de assentamento o alto risco de transmissão da malária é basicamente determinado por variáveis que caracterizam transformações ambientais. Em 1995, dez anos após a abertura do projeto, o perfil de alto risco modificou-se, sendo caracterizado por aspectos econômicos e comportamentais. Segundo, nos anos iniciais do projeto, o perfil de baixo risco está condicionado a aspectos econômicos e comportamentais, em especial à posse de equipamentos (motosserra) e de conhecimento agrícola, o que pode resultar em rápida derrubada e construção de moradia de boa qualidade, reduzindo a exposição ao mosquito transmissor. Terceiro, além de os perfis variarem ao longo do tempo, há também importantes diferenças locais, que não seriam reveladas se o método de GoM tivesse sido aplicado para a totalidade de Machadinho, ressaltando a importância da análise espacial.

Deve-se destacar a necessidade de se incorporarem informações etnográficas e de sensoriamento remoto. Em alguns casos, a descrição de perfis de risco com base apenas em variáveis coletadas na pesquisa de campo não foi capaz de caracterizar corretamente o processo de transmissão. Por exemplo, a presença de lotes onde foi observada significativa transformação ambiental, mas que não foram incluídos na pesquisa de campo por não se enquadrarem na definição de "ocupados", representa um risco adicional que pode ser avaliado apenas através de sensoriamento remoto. A ocorrência de desmatamento ilegal também constitui situação única, que pode ser monitorada por imagens de satélite. De fato, em 1994, uma área de aproximadamente $33,5 \mathrm{~km}^{2}$ foi desmatada a sudeste de Machadinho, tendo empregado mão-de-obra procedente de áreas malarígenas da Amazônia (CASTRO et al., 2006a). O aumento da transmissão de malária nas áreas de Machadinho próximas a essa localidade desmatada não poderia 
FIGURA 3

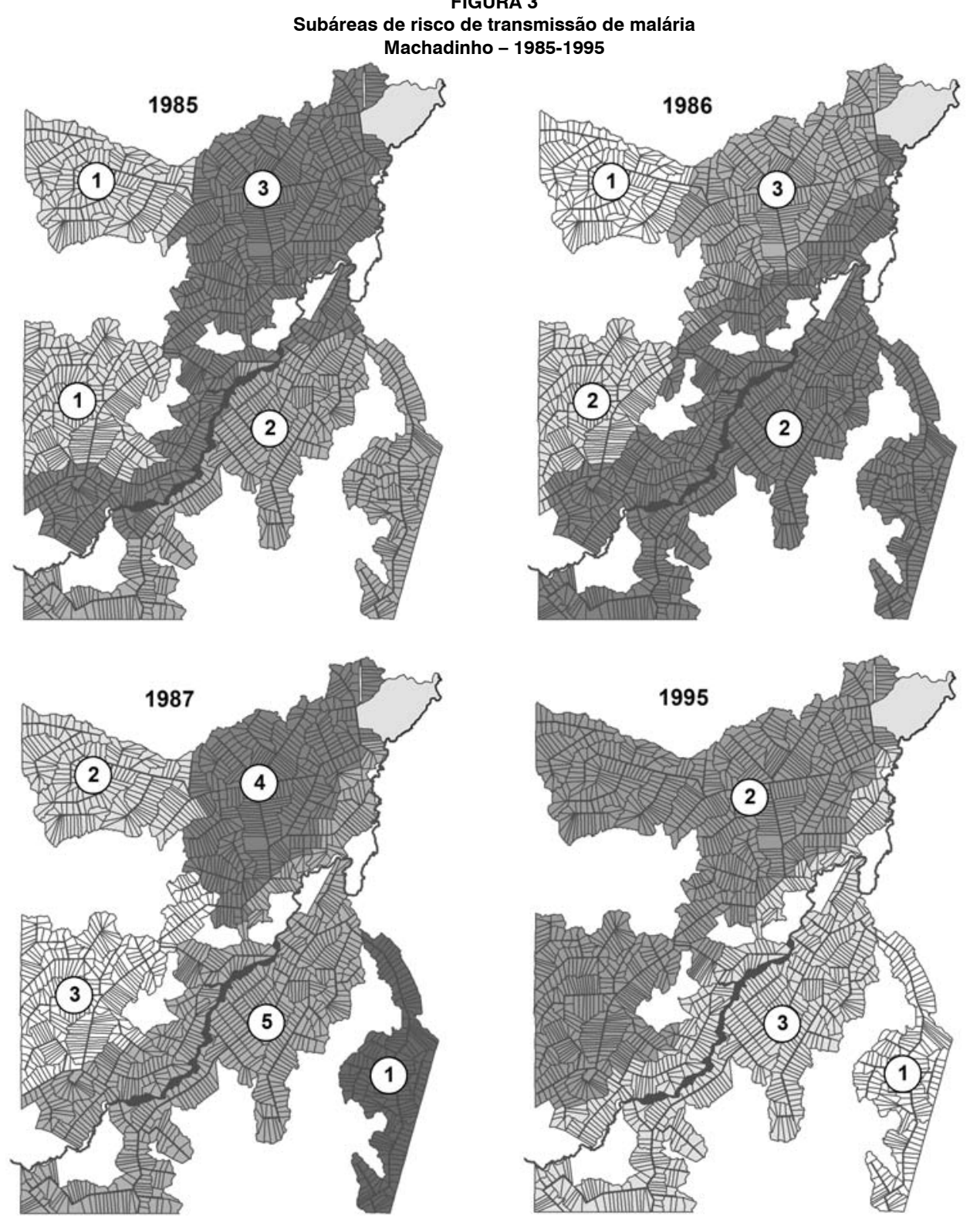

Fonte: Elaboração dos autores.

Nota: Subáreas definidas em cada ano estão indicadas por tons diferentes e por numeração

ser explicado apenas pelos dados coletados na pesquisa de campo.

Com base nesses resultados, algumas medidas mitigadoras assumem importância relevante em projetos de assentamento, tais como:

- com base em estudos de aptidão agrí- cola de solos, alocar parceleiros(as) em áreas que favoreçam a produção de plantações nas quais tenham prévia experiência;

- garantir que o desmatamento inicial para construção de moradia e início de práticas agrícolas seja rápido e 
de boa qualidade e que a moradia ofereça proteção contra o mosquito;

- assegurar assistência técnica em práticas agrícolas;

- iniciar a distribuição de lotes apenas após a instalação de unidades de saúde na área do projeto de assentamento;

- minimizar a presença de casos assintomáticos de malária através do teste laboratorial de novos ocupantes e de grupos populacionais que morem na localidade, tais como seringueiros (CASTRO, 2002; CASTRO et al., 2006a). A primeira e a terceira medidas oferecem condições para práticas agrícolas bem sucedidas, impactando nas condições econômicas do parceleiro e minimizando a chance de abandono do lote. Ainda que estudos de reconhecimento do solo e de aptidão agrícola em Machadinho tenham sido elaborados, tal informação não foi utilizada como critério na distribuição de lotes (EMBRAPA/SNLCS, 1982; CASTRO, 2002). A segunda tem caráter preventivo, reduzindo o tempo de exposição do parceleiro à alta área de risco localizada no limite entre a floresta e a área desmatada, onde o número de criadores de mosquitos é elevado. Finalmente, as duas últimas visam especificamente os serviços de saúde e buscam minimizar as chances de focos epidêmicos da doença. Além disso, postos de saúde poderiam ser veículos de educação sanitária, provendo informações e promovendo o uso de medidas preventivas.

\section{Conclusão}

O entendimento detalhado do processo de transmissão de malária na Amazônia demanda uma análise multidisciplinar, além do uso de metodologia que permita modelar dados heterogêneos. Neste artigo é apresentada uma abordagem metodológica que se destina a esse fim, bem como um exemplo utilizando-se dados do projeto de assentamento de Machadinho, em Rondô- nia. Os resultados revelam importantes diferenças locais e temporais na caracterização de risco e indicam medidas mitigadoras a serem consideradas em projetos de assentamento. Ainda que a implementação de medidas de controle da malária necessite de uma avaliação das características locais de cada área, os resultados aqui expostos aplicam-se de uma forma ampla a outros projetos de assentamento na Amazônia.

Dois aspectos merecem uma discussão mais detalhada. Em primeiro lugar, a abordagem metodológica apresentada não se restringe a estudos de malária. $\mathrm{O}$ mesmo procedimento pode ser aplicado a outras doenças cuja ocorrência seja determinada por múltiplos fatores, tais como dengue, febre amarela, esquistossomose, doença de chagas, entre outras. O importante é que se disponha de dados que permitam avaliar diversas questões potencialmente impactantes no risco de transmissão da doença.

A disponibilidade de dados é a segunda questão que deve ser analisada. As informações disponíveis para Machadinho não possuem a cobertura e a dimensão espacial dos registros oficiais de saúde. Em primeiro lugar, registros oficiais tendem a ser incompletos e enviesados, uma vez que: é comum a inexistência de postos de saúde em áreas pobres e de difícil acesso; a existência de postos de saúde não garante que a população de fato use os serviços, pois a baixa qualidade do atendimento ou a ausência de equipamento resulta na busca por curandeiros ou conselhos em farmácias locais; e a procura por serviços de saúde pode ocorrer apenas quando a doença é de alta gravidade (DE SAVIGNY; BINKA, 2004). Em segundo lugar, registros oficiais podem incluir casos em que o diagnóstico não é correto (por exemplo, casos de febre podem ser diagnosticados como malária sem a comprovação por meio de análise do sangue). Terceiro, esses registros são normalmente indexados pela unidade de atendimento ou município onde foram observados e não pelo local de residência do paciente. Por último, registros oficiais não se equivalem a uma pesquisa de campo e, portanto, não oferecem uma abordagem 
multidisciplinar com relação aos fatores de risco.

Apesar disso, é importante ressaltar esforços positivos que vêm sendo implementados no sentido de melhorar a abrangência e a qualidade da informação, o que eventualmente resultará em melhorias no planejamento das ações de saúde. Por exemplo, em 2003, o Ministério da Saúde aprimorou os dados contidos no Sistema de Informações de Malária (Sismal), a partir da criação do Sistema de Informações de Vigilância Epidemiológica - Malária (SivepMalária) na Amazônia. O Sivep permite rápida entrada e disponibilidade de dados, o que facilita o planejamento e a tomada de decisões. Além disso, a resistência às drogas antimaláricas é monitorada através da Rede Amazônica de Vigilância da Resistência às Drogas Antimaláricas (RAVREDA). Localidades sentinelas foram selecionadas em sete Estados da região, onde a eficácia de medicamentos no tratamento de pacientes é constantemente avaliada (MINISTÉRIO DA SAÚDE, 2006). Essas medidas, ainda que não resolvam problemas de cobertura, contribuem para a melhoria significativa da qualidade dos dados disponíveis e, portanto, da tomada de decisão.

A primeira etapa da abordagem metodológica apresentada neste artigo, aplicada rotineiramente aos registros ofi-

\section{Referências bibliográficas}

AKHAVAN, D. et al. Cost-effective malaria control in Brazil: cost-effectiveness of a malaria control program in the Amazon basin of Brazil, 1988-1996. Social Science \& Medicine, v.49, p.1.385-1.399, 1999.

ALDSTADT, J. et al. Point pattern analysis. Disponível em: <http://www.nku.edu/ longa/ cgi-bin/cgi-tcl-examples/generic/ppa/ppa. cgi>. Acesso em: 22 jan. 2007.

ANSELIN, L. Local Indicators of Spatial Association - LISA. Geographical Analysis, v.27, n.2, p.93-115, 1995. ciais de saúde, representa um sistema de monitoramento espacial da transmissão de malária, apontando a existência de áreas críticas que demandam controle imediato. O SIGEpi, desenvolvido pela Organização Panamericana de Saúde (Opas), ${ }^{2}$ representa um esforço na criação de sistemas de monitoramento utilizando SIG. Considerando a tendência atual de desenvolvimento de programas de análise espacial e geovisualização, em que códigos de programação são disponibilizados a todos os usuários, ${ }^{3}$ a implementação de sofisticados sistemas de monitoramento não demanda elevados investimentos financeiros. O único investimento necessário é capacitação técnica para implementação e manutenção do sistema (CASTRO et al., 2006b).

Outra proposta de uma rotina de vigilância, baseada em medidas de dispersão e métodos de suavização, foi feita por Braz et al. (2006). Tal rotina utiliza os dados oficiais de malária e é de simples implementação. É importante ressaltar, entretanto, que o objetivo maior, reservado para futuros estudos, é a obtenção de modelos preditivos de transmissão de malária, incorporando o caráter multidisciplinar descrito anteriormente e utilizando fontes alternativas de informação. A abordagem metodológica apresentada neste artigo representa um dos passos importantes na consecução deste objetivo.

ARON, J. L.; PATZ, J. Ecosystem change and public health: a global perspective. Baltimore: Johns Hopkins University Press. 2001.

BAUMONT, C. et al. Spatial analysis of employment and population density: the case of the agglomeration of Dijon 1999. Geographical Analysis, v.36, n.2, p.146176, 2004.

BENJAMINI, Y.; HOCHBERG, Y. Controlling the false discovery rate: a practical and powerful approach to multiple testing.

\footnotetext{
2 (http://ais.paho.org/sigepi/index.htm?xml=sigepi/soporte.htm\&lang=en).

3 Alguns exemplos incluem: <http://www.geovistastudio.psu.edu/jsp/index.jsp, http://stars-py.sourceforge.net> e <http:// sal.uiuc.edu/default.php>.
} 
Journal of the Royal Statistical Society B, v.57, n.1, p.289-300, 1995.

BERKMAN, L. et al. Black/white differences in health status and mortality among the elderly. Demography, v.26, n.4, p.661-678, 1989.

BOYD, M. F. Historical review. In: BOYD, M. F. (Ed.). Malariology: a comprehensive survey of all aspects of this group of diseases from a global standpoint. Philadelphia: W. B. Saunders, v.1, 1949, p.3-25.

BRAZ, R. M. et al. Detecção precoce de epidemias de malária no Brasil: uma proposta de automação. Epidemiologia e Serviços de Saúde, v.15, n.2, p.21-33, 2006.

BROWDER, J. O.; GODFREY, B. J. Rainforest cities: urbanization, development, and globalization of the brazilian Amazon. New York: Columbia University Press, 1997.

BRUCE-CHWATT, L. J. Malaria and its control: present situation and future prospects. Annual Review of Public Health, v.8, p.75110, 1987.

CARTER, R. et al. Spatial targeting of interventions against malaria. Bulletin of the World Health Organization, v.78, n.12, p.1401-1411, 2000.

CASTILLA, R. E.; SAWYER, D. O. Malaria rates and fate: a socioeconomic study of malaria in Brazil. Social Science \& Medicine, v.37, n.9, p.1137-1145, 1993.

CASTRO, M. C. Spatial configuration of malaria risk on the Amazon frontier: the hidden reality behind global analysis. (PhD dissertation). Office of Population Research, Princeton University, Princeton, 2002.

CASTRO, M. C. et al. Malaria risk on the Amazon frontier. Proceedings of the National Academy of Sciences, v.103, n.7, p.2452-2457, 2006a.

CASTRO, M. C. etal. Spatial patterns of malaria in the Amazon: implications for surveillance and targeted interventions. Health \& Place, p.Available online 11 July 2006. (DOI 10.1016/j.healthplace.2006.03.006), 2006b.
CASTRO, M. C.; SINGER, B. H. Controlling the false discovery rate: a new application to account for multiple and dependent tests in local statistics of spatial association. Geographical Analysis, v.38, n.2, p.180208, 2006.

CHAGAS, C. Luta contra a malária. Rio de Janeiro, 1934.

COIMBRA JR., C. E. A. Human factors in the epidemiology of malaria in the brazilian Amazon. Human Organization, v.47, n.3, p.254-260, 1988.

COIMBRA, M. E. L. S. SUCAM and malaria control. Belo Horizonte, Cedeplar, 1985.

COVA-GARCIA, P. Notas sobre los anofelinos de Venezuela y su identificación. Caracas: Editorial Grafos, 1961.

CRESSIE, N. Geostatistical analysis of spatial data. In: National Research Council (U.S.). Panel on Spatial Statistics and Image Processing (Ed.). Spatial statistics and digital image analysis. Washington, D.C.: National Academy Press, 1991, p.87-108

CRUZ, O. Madeira-Mamoré Railway Company - Considerações gerais sobre as condições sanitárias do Rio Madeira. Rio de Janeiro, 1910, p.43.

Relatório sobre as condições médico-sanitárias do Valle do Amazonas. Rio de Janeiro, p.111. 1913

DE SAVIGNY, D.; BINKA, F. N. Monitoring future impact on malaria burden in subSaharan Africa. American Journal of Tropical Medicine and Hygiene, v.71 (Suppl. 2), p.224-231, 2004.

DEANE, L. M. Observações sobre a malária na Amazônia brasileira. Revista do Serviço Especial de Saúde Pública, v.1, p.3-60, 1947.

. A história da evolução dos conhecimentos sobre a malária. Rio de Janeiro, 1986, p. 51.

Malaria studies and control

in Brazil. American Journal of Tropical Medicine and Hygiene, v.38, n.2, p.223230, 1988. 
DESOWITZ, R. S. The malaria capers: more tales of parasites and people, research and reality. New York: W.W. Norton, 1991.

EMBRAPA/SNLCS. Levantamento de reconhecimento de média intensidade dos solos e avaliação da aptidão agrícola das terras em $\mathbf{1 0 0 . 0 0 0}$ hectares da gleba Machadinho, no município de Ariquemes, Rondônia. Rio de Janeiro, Embrapa/ SNLCS, 1982.

EROSHEVA, E. A. Bayesian Estimation of the Grade of Membership Model. Proceedings of the Seventh Valencia International Meeting on Bayesian Statistics. Oxford, UK: Oxford University Press, 2003.

FEARNSIDE, P. M. Brazil's Balbina dam: environment versus the legacy of the pharaohs in Amazonia. Environmental Management, v.13, n.4, p.401-423, 1989.

Social impacts of Tucuruí dam. Environmental Management, v.24, n.4, p.483-495, 1999.

GETIS, A. Spatial filtering in a regression framework: examples using data on urban crime, regional inequality, and government expenditures. In: ANSELIN, L.; FLORAX, R. J. G. M. (Eds.). New directions in spatial econometrics. Berlin: Springer, 1995, p.191-203.

GETIS, A.; ORD, J. K.. The analysis of spatial association by use of distance statistics. Geographical Analysis, v.24, n.3, p.189206, 1992.

Local spatial statistics: an overview. In: P. LONGLEY, P. BATTY, M. (Ed.). Spatial analysis: modelling in a GIS environment. Cambridge, New York, 1996, p.261-277.

Seemingly independent tests: addressing the problem of multiple simultaneous and dependent tests. 39th Annual Meeting of the Western Regional Science Association. Kauai, Hawaii, February 26 - March 1, 2000.

GUIMARÃES, A. E. etal. Ecology of Anopheline (Diptera, Culicidae), malaria vectors around the Serra da Mesa Reservoir, State of Goiás, Brazil. 1 - Frequency and climatic factors.
Cadernos de Saúde Pública, v.20, n.1, 2004. p.291-302, jan.-fev.

$\mathrm{HIRSCH}, \mathrm{A}$. Geographical and historical pathology. London: The New Stdenham Society, v.l., 1883.

IÑIGUEZ ROJAS, L. B.; TOLEDO, L. M. D. Espaço \& doença: um olhar sobre o Amazonas. Rio de Janeiro: Ministério da Saúde, Fundação Oswaldo Cruz, Editora Fiocruz, 1998.

ISAAKS, E. H.; SRIVASTAVA, R. M. Applied geostatistics. New York: Oxford University Press, 1989.

JARCHO, S.; TORTI, F. Quinine's predecessor: Francesco Torti and the early history of cinchona. Baltimore: Johns Hopkins University Press, 1993 (The Henry E. Sigerist series in the history of medicine).

KLEIN, T. A.; LIMA, J. B. P. Seasonal distribution and biting patterns of anopheles mosquitoes in Costa Marques, Rondônia, Brazil. Journal of the American Mosquito Control Association, v.6, n.4, p.700-707, 1990.

KURTZ, T. E. et al. Short-cut multiple comparisons for balanced single and double classifications: Part 1, Results. Technometrics, v.7, n.2, p.95-161, 1965.

LOURENÇO-DE-OLIVEIRA, R. et al. Anopheline species, some of their habits and relation to malaria in endemic areas of Rondônia State, Amazon region of Brazil. Memórias do Instituto Oswaldo Cruz, v.84, n.4, p.501-514, 1989.

MANTON, K. G. et al. Statistical applications using fuzzy sets. New York: J. Wiley, 1994

MARQUES, A. C. Human migration and the spread of malaria in Brazil. Parasitology Today, n.3, p.166-170, 1987

MARTINE, G. Rondônia and the fate of small producers. In: GOODMAN, D.; HALL, A. L. (Eds.). The future of Amazonia: destruction or sustainable development? Houndmills, Basingstoke, Hampshire: The Macmillan Press LTD., 1990, p.23-48. 
MINISTÉRIO DA SAÚDE. Situação epidemiológica da malária no Brasil. Brasília, DF, Ministério da Saúde, Secretaria de Vigilância em Saúde, Diretoria Técnica de Gestão, 2006.

MORAN, E. F. An assessment of a decade of colonization in the Amazon Basin. In: HEMMING, J. (Ed.). Change in the Amazon Basin: the frontier after a decade of colonization. Manchester: Manchester University Press, v.ll, 1985, p.91-102.

NÁJERA, J. A. et al. Malaria: new patterns and perspectives. Washington, D.C.: World Bank, 1992 (World Bank technical paper, n. 183).

ORD, J. K.; GETIS, A. Local spatial autocorrelation statistics: distributional issues and an application. Geographical Analysis, v.27, n.4, p.286-306, 1995.

PAEZ, A. et al. Spatial association and heterogeneity issues in land price models. Urban Studies, v.38, n.9, p.1493-1508, 2001.

PEIXOTO, A. O problema sanitário da Amazônia. Rio de Janeiro, 1917.

ROGERSON, P. A. A statistical method for the detection of geographic clustering. Geographical Analysis, v.33, n.3, p.215227, 2001.

SAWYER, D. R. Research design and feasibility in the Machadinho settlement project. Belo Horizonte, Cedeplar, 1985.

. Frontier malaria in the Amazon region of Brazil: types of malaria situations and some implications for control. Brasília, PAHO/WHO/TDR, April, 1988.

Malaria and the environment. Brasília, Instituto SPN, March, 1992.

SAWYER, D. R.; SAWYER, D. O. Malaria on the Amazon frontier: economic and social aspects of transmission and control. Belo Horizonte, Cedeplar, 1987.

The malaria transition and the role of social science research. In: CHEN, L. C. (Ed.). Advancing the health in developing countries: the role of social research. Westport: Auburn House, 1992, p.105-122.

SCHMINK, M. Social change in the garimpo. In: HEMMING, J. (Ed.). Change in the Amazon Basin: the frontier after a decade of colonization. Manchester: Manchester University Press, v.ll, 1985, p.185-199.

SCHMINK, M.; WOOD, C. H. Contested frontiers in Amazonia. New York: Columbia University Press, 1992.

SILVA, G. D. C. E. Geopolítica do Brasil. Rio de Janeiro: José Olympio, 1967 (Coleção Documentos Brasileiros, 126).

SILVEIRA, A. C.; REZENDE, D. F. D. Avaliação da estratégia global de controle integrado da malária no Brasil. Brasília, Organização Pan-Americana da Saúde, 2001.

SINGER, B. H. Grade of membership representations: concepts and problems. In: KARLIN, S. et al. (Eds.). Probability, statistics, and mathematics: papers in honor of Samuel Karlin. Boston: Academic Press, 1989, p.317-334.

SINGER, B. H.; CASTRO, M. C. Agricultural colonization and malaria on the Amazon frontier. In: WEINSTEIN, M.; HERMALIN, A.; STOTO, M. A. (Eds.). Population health and aging: strengthening the dialogue between epidemiology and demography. New York: Annals of the New York Academy of Sciences, v.954, 2001, p.184-222.

SINGER, B. H.; SAWYER, D. O. Perceived malaria illness reports in mobile populations. Health Policy and Planning, v.7, n.1, p.4045, 1992.

SYDENSTRICKER, J. M. Parceleiros de Machadinho: história migratória e as interações entre a dinâmica demográfica e o ciclo agrícola em Rondônia. Dissertação de mestrado. Campinas, Universidade de Campinas, 1992.

TADEI, W. P. et al. Biologia de anofelinos amazônicos. XII. Ocorrência de espécies de anopheles, dinâmica da transmissão e controle da malária na zona urbana de Ariquemes (Rondônia). Revista do Instituto 
de Medicina Tropical de São Paulo, v.30, n.3, p.221-251, 1988.

TADEI, W. P. et al. Ecologic observations on anopheline vectors of malaria in the brazilian Amazon. American Journal of Tropical Medicine and Hygiene, v.59, n.2, p.325335, 1998.

TOBLER, W. R. Cellular geography. In: GALE, S.; OLSSON, G. (Ed.). Philosophy in geography. Dordrecht: D. Reidel Pub. Co., 1979, p.379-386.
TUKEY, J. W. The philosophy of multiple comparisons. Statistical Science, v.6, n.1, p.100-116, 1991.

VAN THIEL, P.H. Malaria problems arising from the construction of a reservoir in the interior of Surinam. Tropical and Geographical Medicine, v.14, p.259-278, 1962.

WAGLEY, C. Amazon town: a study of man in the tropics. New York: Alfred A. Knopf, INC., 1964, xi; 315; vi p.

\section{Resumen}

Medio ambiente y salud: metodología para análisis espacial de la ocurrencia de malaria en proyectos de asentamiento

La transmisión de malaria en proyectos de asentamiento en la Amazonia, definida como malaria de frontera, es el resultado de un intrincado proceso, involucrando factores biológicos, ecológicos, socioeconómicos y comportamentales, presentando una transición temporal de altas a bajas tasas a lo largo de aproximadamente ocho años. Como resultado, uno de los grandes desafíos es la comprensión de ese proceso, a través de la identificación de las variables determinantes de la transmisión, habiéndose considerado dimensiones temporales y espaciales. En este artículo es presentado un abordaje metodológico que caracteriza perfiles de riesgo de malaria en proyectos de asentamiento, a partir de un análisis multidisciplinario. Compuesto de tres etapas, el abordaje combina análisis espacial, geoestadística y modelos de Grade of Membership. Los resultados resaltan la importancia de medidas de control diferenciadas, de acuerdo a la etapa del proyecto de asentamiento (implementación reciente o antigua) y al nivel de transmisión en cada localidad.

Palabras-clave: Malaria. Proyectos de colonización. Amazonia. Krigaje. Indicadores locales de asociación espacial. Modelos de Grade of Membership.

\section{Abstract \\ Environment and Health: a methodological approach for spatial assessment of malaria transmission in colonization projects in the Brazilian Amazon}

Malaria at colonization sites in the Brazilian Amazon is defined as frontier malaria, a complex phenomenon including biological, ecological, socioeconomic, and behavioral issues. It follows a specific temporal transition cycle lasting approximately eight years, oscillating from extremely high rates of transmission to lower and stable ones. A broader understanding of this phenomenon that would account for its spatial and temporal idiosyncrasies is a major challenge. In this article we present a methodological approach that describes malaria risk profiles based on a multidisciplinary analysis. The approach combines spatial analysis, geostatistical tools, and fuzzy-set models. Results highlight the need for spatially and temporally targeted interventions for mitigating the spread of this disease.

Keywords: Malaria. Colonization projects. Amazon. Kriging. LISA. Grade of Membership models. 